\title{
MAGNETIZED ACCRETION DISKS DRIVING JETS
}

\author{
G. PELLETIER, J. FERREIRA and F. ROSSO \\ Laboratoire d'Astrophysique de l'Observatoire de Grenoble \\ Domaine Universitaire. BP 53X. 38041 Grenoble. France
}

\section{Framework}

In this brief communication, we present some progress in the investigation of a most promising model that was designed to combine ejection with accretion. In this model, a bipolar configuration of opened magnetic field lines that thread the accretion disk, allows the extraction of angular momentum, the acceleration of matter up to super Alfvénic velocities and the self collimation of the jet. However, important issues have remained unsolved. First, a systematic method for solving the jet MHD equations with their critical surfaces was lacking. Second, the capability of accretion disks to generate super Alfvénic jets was unknown. Third, the back-reaction of the ejection on the accretion disk dynamics and its energetics remained to be done. Solving these three points led us to draw some noteworthy consequences for the understanding of AGNs.

\section{Jets from quasi Keplerian disks}

Solving for the jet MHD equations is very difficult either analytically or numerically (Lovelace et al. 1987), because of the existence of unknown critical surfaces, mostly Alfvén (A) surface and fast magnetosonic (FM) surface, and of unknown surfaces where the PDEs change their type (from elliptical to hyperbolic and vice versa). These difficulties were rounded (Rosso and Pelletier 1993) by using a functional, whose extremalization with respect to the local Alfvén Mach number $m$ and to the flux function $\psi$ leads to the equations of Bernoulli and Grad-Shafranov. This functional reads:

$$
\int\left(\frac{1}{2} \frac{m^{2}-1}{r^{2}}(\nabla \psi)^{2}+U\left(m^{2}, \psi ; r, z\right)\right) r d r d z
$$

So the solution of the problem was obtained by extremalizing the functional under the conditions for the flow to pass through the critical surface and to satisfy regularity conditions, and also to match a boundary condition at the accretion disk surface. Because of the dominent Keplerian motion, some self-similarity properties of the accretion disk hold on a wide radius interval. This implies the same similarity to the outflow, which is exactly admitted by the MHD equations for the jet. These classes of self-similar solutions contains Blandford and Payne (1982) and Konigl (1989) solutions as particular solutions. We obtained a flow diagram on a given magnetic surface that revealed that the Alfvén points are bifurcation points where three types of flow can be discriminate: a sub-A flow, a super-A but sub-FM flow, and a super-FM flow.

T. J.-L. Courvoisier and A. Blecha: Multi-Wavelength Continuum Emission of AGN, 249-252. (C) 1994 IAU. Printed in the Netherlands. 
Three noteworthy results were obtained. First, as we considered jets carrying a poloidal current, we confirmed the result, obtained by Heyvaerts and Norman (1989), that the jet undergoes cylindrical self-collimation after having passed through the critical surfaces. A return current inside the jet itself would cancel the self- collimation (Lovelace et al. 1991). Second, whatever the enthalpy in the disk corona, significant bending is required to get a jet that crosses the A and FM surfaces. We know from Blandford and Payne (1982) that a cold outflow from a disk can become a super- $A$ jet provided that the field lines bend more than $30^{\circ}$ from axis at the surface of the disk. However a wind can be launched from the disk even if the field lines are straight, provided that a corona give it enough enthalpy. But our results show the requirement of an important bending to get a super-FM jet. Third, despite the magnetic surface widening above the accretion disk, the centrifugal acceleration does not lead to asymptotic velocities much larger than the Keplerian velocity at the foot of the field lines because it turns out that the A-surface is close to the accretion disk. We have explored a wide range of parameters and we did not find any situation where the A-surface is remote. So jets from quasi keplerian accretion disks are more likely subrelativistic. We think that the only possibility to launch relativistic jets is in the vicinity of a fastly rotating black hole (Blandford and Znajek 1977, Camenzind 1986) and probably in the form of relativistic flow of electrons and positrons (Baskin et al. 1991). However it is not yet sure that the Compton drag can be overcome. Alternately, a model of relativistic ejection of $e^{+} e^{-}$pairs that starts between 50 and $100 r_{G}$ from the black hole, a distance where Compton drag is weakened, was proposed (Henri and Pelletier 1991). This ejection, that is powered and channeled by the subrelativistic disk outflow, takes the form of a continuous or sporadic relativistic beam and likely explains the high energy spectrum of radio quasars (Henri et al.1992, Henri and Markowith 1993).

Is it possible for an accretion disk to display such bended open field lines?

\section{Accretion disks revisited}

R. Pudritz (1986) proposed that an MHD jet could extract the angular momentum of the accretion disk and thus makes the turbulent viscosity useless. In this scheme, the jet controls the accretion process and properties of such a jet was derived (Pelletier and Pudritz 1992). Indeed the most powerfull jets are those that extract most of the angular momentum and thus modify significantly the accretion disk properties. Our first attempts (Ferreira and Pelletier 1993a) to solve completely the MHD equations for a magnetized accretion disk with no viscosity succeeded to give a solution where the MHD wind extract completely the angular momentum, but with bipolar straight field lines. In this particular configuration, all the gravitational power is stored in the magnetic field, the disk emission being just a secondary process. Thus, the luminosity is not a measure of mass flux and Eddington estimates are merely fiducial numbers. We expected that the magnetic surfaces would widen at a remote distance, so that the wind could become super 
Alfvénic; but our previously mentioned results about jets indicate that this is not possible. New attempts (Ferreira and Pelletier 1993b), including a weak viscous effect, led to successful solutions with a significant bending of the field lines. An interesting class of solutions was studied with more details because all the involved physical effects can be put together on a wide interval of radii of the disk. This is for a flux distribution on the midplane such that $\psi \propto r^{-3 / 4}$. Hence we solved the complete problem, including the vertical structure, with i) angular momentum extraction by both magnetic and viscous torques, ii) heating by both Joule and viscous dissipation, iii) vertical equilibrium by both magnetic tension and gravitation compression. Even for a magnetic torque 50 times larger than the viscous torque, we obtained jet solutions with field lines bended of $50^{\circ}$ from axis, provided than the magnetic Reynolds number be sufficiently larger than unity (of order 10). Indeed the more the magnetic Reynolds number $\mathcal{R}_{m}$, the more the field lines bending. In the evaluation of $\mathcal{R}_{m}$, defined by

$$
\mathcal{R}_{m} \equiv \frac{r u_{r}}{\nu_{m}}
$$

we need an anomalous magnetic diffusivity $\nu_{m}$ such that $\nu_{m}=\alpha_{m} V_{A} h$, in order to have a stationary flow of matter crossing the magnetic surfaces with a thin disk configuration. This is required by steady MHD equations; moreover the number $\alpha_{m}$ must be of order unity to quench resistive instabilities, because it is the ratio of the Alfvén time across a disk of thickness $h$ over the resistive time for the same scale. Increasing $\mathcal{R}_{m}$ by reducing $\alpha_{m}$ leads to convection instabilities by producing superadiabatic gradients and resistive instabilities. Inside the disk the resistive instability is expected to produce magnetic islands by field line reconnections. But this occurs under conditions where the internal pressure plays an important role. This is not the case in solar corona nor in tokomaks; and so the dynamics of reconnections in high $\beta$ plasmas is not really known. However we could expect that the reconnections occuring in the disk corona where the magnetic configuration is almost force free are similar to those of the solar corona. In fact, the microturbulence generated along the neutral lines of reconnections can bring the amount of anomalous resistivity needed, and moreover the electric fields along the neutral lines are likely to generate the relativistic electrons responsible for the high energy spectra.

The magnetic field reroutes part of the stream lines in the accretion disk, even in the optically thick region; they turn to more than $120^{\circ}$ and tends asymptotically towards the field line outside the disk, achieving an almost ideal MHD flow, at few disk thicknesses, with a magnetic Reynolds number of order $10^{3}$. All these classes of self-similar solutions for the disk henforce to the same similarity for the jet solutions, because they correspond to exact similarity properties of the MHD equations. So the matching between the accretion disk and the ideal MHD jet is realised, at least for some parameter range. We got these results even under conditions where the magnetic pressure is smaller than the radiation pressure. 


\section{Synthesis for AGNs}

Our view of AGNs exhibiting jets can be summarized as follows. Three types of situations can be discriminated, likely due to the conditions of magnetization of the accretion disk.

i) In AGNs with FR I radiojets, the jets seem clearly non relativistic and their production by a quasi Keplerian accretion disk is very likely. However, some relativistic outburts can occur (in Bl Lac, for instance).

ii) In AGNs with FR II radiojets, large scale structures and hot spot parameters do not dictate relativistic motions (Roland et al. 1988) (Meisenheimer et al. 1989). Relativistic velocities are only clearly suggested by the superluminal motions of VLBI knots. We consider that the accretion disk can power most of the ejection in the form of a mildly or sub relativistic MHD flow. The relativistic ejection would come from a beam (Sol et al. 1988), either from a fastly rotating black hole if Compton drag is really overcome, either from pair creation at the edge (50 to 100 $r_{G}$ ) of the "magnetized cauldron" (Henri and Pelletier 1991).

iii) In AGNs of Seyfert galaxies, the outflow from the accretion disk does not pass through the critical surfaces, but some possibility of an aborted jet exists; which is convenient to explain the high energy spectrum.

Indeed, in the three cases, we propose that a magnetic cauldron made by the MHD outflow, heated by Alfvén turbulence, contains a plasma of relativistic pairs at a decentred location, illuminating the disk with hard X-rays and interacting with the hot absorbing medium of the subrelativistic outflow (See Ferreira et al.. 1994). The results we obtained in the framework of a quasi Keplerian disk driving MHD jets are encouraging to understand the main energetics of the accretion-ejection process and deserve deeper physical investigations, especially regarding instabilities and transport phenomena.

\section{References}

Beskin V.S., Istomin Y.A., Pariev V.I. (1991), "Extragalactic Radio Sources", proc. 7th IAP meeting, Paris 1991.

Blandford R.D. and Payne D.G. (1982), MNRAS, 199, 883

Blandford R.D. and Znajek D.G. (1977), MNRAS, 179, 433

Camenzind M. (1986), A. and A., 156, 137

Ferreira J. and Pelletier G. (1993a), A. and A., 276, 625 and A. and A., 276, 637

Ferreira J. and Pelletier G. (1993b), A. and A.,(submitted)

Ferreira J., Henri G., Markowith A., Pelletier G., Rosso F. (1994) A. and A. (in preparation)

Henri G. and Pelletier G. (1991), ApJ Letter, 383, L7

Henri G., Pelletier G., Roland J. (1992), ApJ Letter, 404, L41

Henri G. and Markowith A. (1993), proceeding of the conference

Heyvaerts J. and Norman C. (1989), ApJ, 347, 1055

Konigl A. (1989), ApJ, 342, 208

Lovelace R.V.E., Wang J.C.L., Sulkanen M.E. (1987), ApJ, 315, 504

Meisenheimer K., Roser H.J., Hiltner P.R., Yates M.G., Longair M.S., Chini R., Perley R.A. (1989), A. and A., 219, 63

Pelletier G., Pudritz R.E. (1992), ApJ, 394, 117

Pudritz R.E. (1986), ApJ, 301, 571

Roland J., Pelletier G., Muxlow T.W.B. (1988), A. and A., 207,16

Rosso F. and Pelletier G. (1993), A. and A., (submitted)

Sol H., Pelletier G., Asséo E. (1989), MNRAS, 237, 411 